the anatomical relations of the parts, that before either, and especially the latter, can take effect an enlargement of the hypophysis must already project some distance above the normal level of the diaphragma sellæ, according to Hirsch, at least $7.5 \mathrm{~mm}$. and, according to Cope, $10 \mathrm{~mm}$. or more. There is no evidence to show that the earliest field changes are delayed until such dimensions have been attained by the tumour.

(To be concluded.)

\title{
SOME PHASES OF MODERN OCULAR THERAPEUTICS.*
}

\author{
BY \\ The late Wendell Reber, M.D., \\ PHILADELPHIA, PA., U.S.A.
}

Since the days of the Ptolemies, the medical man with his healing art has been an integral part of civilization. For centuries the human race was satisfied with mysticism and occultism. But a more dignified position was given the healing art by the ancient Greeks, among whom there were three schools or sects in medicine. First: the empirics who insisted that experiment was the prime requisite. Second: the methodists who fell back upon theory. Third: the dogmatists who took middle ground.

In more recent times, experiment and experience have been relied upon as essentials in medicine, and especially that part of it which concerns therapeutics. We all concede that an ounce of prevention is worth a pound of cure. But human life is so inextricably bound up with what Samuel D. Risley has happily called "the physiologic vices" of civilization that preventive medicine, with all its boasted achievements, affords little prospect of doing away with the healing art, at least in our day and generation. So that the cure of disease is still our sacred function. Except we heal our patients, our science and art is in vain. We are become as sounding brass and tinkling cymbals. If we cannot prevent, we must cure the ills that flesh is heir to. Therapy, therefore, must and will remain the supreme eftort in combating disease.

The empirical nature of nearly all of our best methods and remedies has been often recognised, and even to-day it is surprising to what an extent we still depend upon what can be credited to empiricism and this alone. I would not for a moment disparage scientific medicine nor the desire to place all medical knowledge

\footnotetext{
*Address delivered by invitation before the'New England Ophthalmological Society, December 7th, 1915.
} 
on a sound scientific basis. But to wait until a remedy or measure is scientifically established before taking advantage of the benefits that experience or observation have taught us to expect from its use, is not only unjust to our patients, but is a violation of that humanity which is the tap root of our art and profession.

The word "incurable" should be the most dreadful of nightmares to a medical man ; and even the utmost pessimist among us must grudgingly admit his conviction that eventually the most redoubtable diseases will little by little find their prophylaxis or their remedy. Calomel, that gift of the gods, has been used for centuries, and even yet the laboratory workers have not told us its exact mode of action. Pilocarpin has been known as a general lymphagogue and eliminant for forty years, but only in the last five years have the serologists offered an explanation of its action that really explains. And so I plead for the open mind, that without haste and without rest is willing to prove all things. The consulting-room of each and everyone of us becomes a laboratory of experimental therapeutics if the observations there made are controlled and properly interpreted. And if perchance there should arise a conflict in testimony between the test-tube result in the laboratory and the results of carefully conducted therapeutic tests in the consultingroom or clinic, my voice would be raised in favour of the latter until such time as the results can be harmonized. For not always are the biologic conditions that obtain in the eye faithfully duplicated in the test-tube. Hence, the wisdom of reserving final judgment until the academic and the actual can be made to fit into each other.

It is a far cry from the atropine, silver, and mercury of the days of von Arlt to the embarrassment of therapeutic riches that encompasses us on every hand to-day. It may be well, therefore, to review, to take stock, as it were, of our armamentarium, and to learn, if so be, which are our most valued weapons.

With these brief words of introduction I shall proceed to the main topic of our discussion, and shall analyze certain phases of ocular therapeutics according as they are employed for (1) local use in the eye only, and (2) as a combination of general and local measures.

\section{Local remedies and measures.}

From 1833, when Geiger succeeded in extracting atropine from the Solanacea, this alkaloid was supreme in the domain of ophthalmology for 50 years. That it was abused goes without saying; indeed, many an ophthalmologist's title was based on no more substantial foundation than that he employed atropine in his practice. In 1885, Koller introduced cocain, to be followed by its congeners, eucain, beta-eucain, holocain, novocain, stovain, alypin, 
acoin, and a host of others, each with its own particular champion. Of them all, cocain still seems supreme as the anæsthetic of election in major ophthalmic surgery. Unfortunately, because of its loose chemical constitution, it cannot be boiled without losing much of its prized anæsthetic properties, and certain well-known authorities have therefore renounced it in favour of holocain or novocain, both of which may be boiled in solution, and thus sterilized, without impairing their special properties. Holocain, however, deserves more than passing notice, because of its beneficent influence upon all stages of corneal ulcer, a point that is not always sufficiently taken advantage of.

Not least among the synthetics with which organic chemistry has flooded us is dionin. It is almost gratuitous to observe that there has been much conflict of opinion as to the value of this drug. My own experience is that it is of signal service in promoting the absorption of post-operative débris. That it distends the conjunctival lymphatics from 5 to 10 times their normal dimensions seems reasonably well proven, and this may serve to explain its helpful action in iritis and uveitis. Also, as I. pointed out ten years ago, in those rare cases of iritis glaucomatosa (Goldzieher) which present sooner or later to all of us, it is the ideal therapeutic "straddle." It is the one drug that seems equally efficacious in either iritis or glaucoma, and in its own domain stands alone. We should consider our own treatment case incomplete without it.

Next among the synthetics must be mentioned the various combinations of protein with silver, the so-called organic silvers. Whether one is wedded to protargol, argyrol, argentos, collargol, argentamin, largin, silvol, or whatever other preparation may be mentioned, matters little. The outstanding fact is that inorganic nitrate has been largely displaced by the organic silvers, and justly so. In acute infections of the anterior ocular segment their efficacy is beyond dispute, if only the precaution is observed never to continue their use over two or, at the most, three weeks. I am strongly of the belief that more argyrosis can be laid at the door of the organic silvers than ever obtained with the nitrate in its palmiest days. Albeit, we have finally elected argyrol as the least irritant of them all. For eight years, following the suggestion of Henry Dickson Bruns, of New Orleans, it has been our invariable practice to introduce argyrol in 25 per cent. solution into the conjunctival sac just before closing the eye after all major operations, and in that time we have had but one purulent post-operative infection. It is also used at each subsequent dressing, for at least ten days to two weeks. We concede that many other operators have achieved 'quite as fine results without resorting to this agent, but this in nowise disturbs the confidence we repose in argyrol as a preventive of post-operative infection. Not least among the advantages of this 
method is the beautifully dry eye, free from any secretion, that is found each time the eye is redressed. In the ophthalmias, specific and non-specific, we lean very heavily upon 25 to 40 per cent. solution of this agent, although the inorganic nitrate is still a sovereign agent, when properly used.

Of most recent introduction is the new cinchona derivative, ethylhydro-cuprein, or "optochin," if the shorter trade name is preferred. In pneumococcus infections of the conjunctiva and cornea this new synthetic gives much promise. In the conjunctival affections its action is prompt and lasting, although too much insistence cannot be laid upon the imperative necessity of fresh solutions. Not more than a 24 or at the most 48 hour supply should be ordered at one time, for like all the photo-chemical synthetics, it deteriorates rapidly. In the few corneal cases that we have studied its action, it has seemed of service.

There is another field in which it may play no inconsiderable part, namely, as an anæsthetic. Like acoin, it exerts a much more anæsthetic effect when introduced subconjunctivally, and if the French reports are borne out, the anæsthesia should be of 8 to 12 hours' duration. This would be ideal in muscular operations as done under local anæsthæsia, for if the parts can be kept anæsthetic for twelve hours after the operation, it will much assist the quiet that is so essential after advancement operations. I have used it this way in but one advancement operation, and the anæsthesia endured for the best part of 24 hours afterwards. Not more than $\frac{1}{2}$ per cent. solutions should be used subconjunctivally, as it seems disposed to favour sloughing in stronger solutions.

\section{Subconjunctival injections.}

Local therapeusis is now the order of the day, not alone in ophthalmology but in all the branches of medicine. Whenever it is possible to strike at a morbid focus of disease, the surgeon lays claim to a considerable share in the treatment of maladies once supposed to belong exclusively to the domain of medicine. Darier has well said, "Whenever a primary or secondary affection is localised in an organ as important as the eye, it becomes of the greatest consequence to prevent and extinguish the infection locally if possible without losing sight of the general indications. Now the eye lends itself beautifully to local therapeutics because of the arrangement of its lymphatic system which is made up of spaces communicating readily with one another." On this basis we can explain the favourable action of normal saline solutions given by subconjunctival injection. To promote absorption of recent corneal opacities, to stimulate absorption of vitreous opacities, also deposits upon the anterior capsule in the later stages of uveitis, we would award it a high place. All told, we have made 
at least 1,000 such injections with various therapeutic ends in view without observing any untoward results. A few scattered cases of glaucoma have come to report alleged to have resulted from subconjunctival injections of normal saline solutions, but their number is negligible. Moreover, they may have occurred because the injection was not done as near the equator of the eyeball as possible. As Darier as shown, this is essential. For detachment of the retina we know of no measure as promising of results. We have now under observation a 55 year old man with spontaneous bilateral detachment, who 15 months ago had vision of $1 / 200$ in his best eye. Injections of saline solution, running up to 10 per cent. strength, have been made three to four times monthly during the 15 months and to-day his vision in that eye is 6/12. For six months he has been going about actively every day. One such result in so dismally hopeless a disease as retinal detachment will outbalance a whole volume of negative records. Citrate of soda solutions have not afforded me entire satisfaction. To combat ocular infections, injections of 1 to 1,000 solutions of bichloride of mercury have proven their worth. In my service at the Philadelphia General Hospital we have a large number of hypopyon-ulcers of the cornea to deal with. Invariably they are treated with 1 to 1,000 solution of bichloride subconjunctivally and the results far outrun any others we have witnessed. Occasionally a second injection is necessary but not often.

Oxycyanide of mercury solutions need greater dilution, 1 to 5,000 to 1 to 8,000 solutions being preferred. This solution we have reserved for choroidal lesions, and we can attest its worth in such lesions, if recent and not due to tuberculosis. It is well in such injections to resort first to the customary cocain anæsthesia (four instillations of a 4 per cent. solution) followed by three to four minims of 1 per cent. acoin solution; five minutes after which 15 to 20 minims of the mercurial agent are introduced. Serum gelatine acoin (prepared by M. Peliolle, a pharmacist, of Poste St. Denis, Paris) has proven most acceptable for this purpose. To sum up this phase of the subject, we may claim that "with solutions of sodium chloride of different degrees of concentration, we can provoke a powerful phagocytic, lymphagogue, eutrophic, and exosmotic action, while with the mercurial solutions we can set up antiseptic resolutive and antisyphilitic effects of a pronounced kind."

Some have claimed that the various serums may be profitably employed subconjunctivally, and that they have the advantages possessed by all local intervention. Hyperemia of the ciliary body is thus induced, and Wessely holds that a more copious afflux of antibodies into the aqueous humor results. Römer's method is to obtain the serum from a blister artificially produced, and he reports 40 cases of hypopyon-keratitis in which most excellent results are claimed. It is significant, however, that evacuation of the hypopyon 
surgically was done in each case, which, to our way of thinking, would have probably effected a cure without the use of the serum. We shall have more to say about this method of autoserotherapy later on.

\section{Drugs and other substances for internal administration.}

For a long time it has seemed to me that the supreme therapeutic test of an ophthalmologist is his facility and resourcefulness in the internal administration of drugs or other substances for the cure of ocular diseases. If we go back to the Fathers of Ophthalmology we shall be at once impressed with their familiarity with general medical problems and principles. Many of them served a long apprenticeship in general medical service before restricting themselves to the consideration of ophthalmic problems only. And that it was to the unending and incalculable profit of their patients and of medical and ophthalmic science, no one can gainsay. I do not wish to labour the point, but I cannot here refrain from setting down my conviction that the ophthalmologist who is not first and foremost a medical man is a precious poor ophthalmologist. Our profession to-day offers numerous instances of brilliant but fearfully one-sided men whose viewpoint is shockingly distorted because of a lack of that broad medical foundation that is the starting point of all medical science. Witness, for instance, the indiscriminate use of mercury and the iodides. On such flimsy foundations are therapeutic diagnoses frequently made. Sometimes a parenchymatous keratitis is cured by mercurial treatment, and later the tuberculous origin of the disease becomes manifest. We should no longer be contented with symptomatic treatment, but delve into every corner in the search for the true xtiology. Let us grant, however, that we cannot always have an exact ætiologic basis for our general therapeusis and pass on to the consideration of the general means and measures at our command.

The doctrine of ascending doses of drugs is as old perhaps as the healing art. Is it as often taken advantage of as it should be? I doubt it. In the matter of the iodides, of Fowler's solution, and of dionin, it is, of course, in general use. But is it true of nux vomica and strychnia? In certain forms of muscular insufficiency, notably that of convergence, as suggested by de Schweinitz, this method has often rendered me yeoman service. And in the phlyctenular disorders of children, syrup of iodide of iron in ascending doses seems to me the most valuable single agent we possess. One of my little six year old patients is now taking 50 drops of the syrup three times a day and is flourishing to a degree. In the interstitial keratitis of childhood, it is also almost a panacea, and one of my ten year old patients recently took up to 90 drops of the syrup three times daily with almost magical results. Thyroid substance, thymus substances, 
and pituitary substance are all of them vastly more effective if so employed. The same holds true of local remedies. Atropine gradually loses its hold in long-standing iritis, and nothing is of commoner observance than the diminishing effect of the same strength of solutions of the miotics in chronic simple glaucoma. One old lady with this malady is now using a $5 \mathrm{gr}$. to the ounce solution of eserin salicylate in her eyes three times a day. She began with a $1 / 4 \mathrm{gr}$. to the ounce solution of the drug and has been 10 years advancing to the present dose, but her vision remains $6 / 12$ in one eye and $6 / 6$ in the other, and her visual fields are fully preserved. There are many who would argue. that if the dear old lady had been operated on ten years ago there would have been no need of all this ten years of constant attention, but in this particular connection I cannot repress the temptation to quote from the small boy's essay on pins, wherein he said, "Pins have saved many people's lives by not swallowing them!" The moral is obvious.

In short, this axiom of the need of ascending doses of drugs is so palpable that it seems almost gratuitous to bring it to notice before this distinguished body, and I should have omitted it were I not persuaded that it is honored more frequently in the breach than in the observance.

Next we come to the consideration of a potent drug that has been more enthusiastically acclaimed and more bitterly assailed than almost any other in our whole armamentarium. I refer to pilocarpin. That it is not without its dangers no conscientious therapist will deny. In organic disease of the heart it is generally contraindicated, and there are a few other conditions in which it is not well borne. But given the proper indications it is the eliminant par excellence. It has been urged that mechanical diaphoresis by arousing the superficial lymphatics accomplishes all that pilocarpin can be hoped to do without the inconveniences and latent dangers of the latter. To refute this postulate I am fond of referring to the hydra, a small aquatic animal sometimes found in aquariums. Its. structure is extremely simple in that it consists of a series of rings of tissue so united that the animal is in reality a hollow tube. The most interesting fact about this tiny creature is that it may be readily turned inside out without in the least disturbing its functions. Immediately what was its mucosa becomes its skin and what was. its skin becomes its mucosa. Without any evidence of incon,venience, it pursues the even tenor of its way. And if one choose one can again turn the creature inside out, when once more the animal promptly adapts itself to the reversed anatomy. Once more its skin becomes its gastro-intestinal tract and its gastro-intestinal tract its skin, and the little mariner goes on its way happy and contented. Such adaptation to environment is, of course, beyond human accomplishment, and it may be a far cry, but I am going to assume 
for the sake of illustration that we are but glorified and complex hydras. The inter-relation of our skins and our gastro-intestinal tracts hardly needs mention here were it not that pilocarpin not only arouses the cutaneous expanse, but also the whole mucosa from the mouth to the anus. Not only that, but some of the viscera respond to its stimulus, and recently laboratory investigation has credited to pilocarpin qualities far beyond the most extravagant claims that have ever been made for it by clinicians. I quote from that recent remarkable book by Kolmer, entitled: "Infection, Immunity and Specific Therapy,"* Kolmer states (page 157) as follows: "In reality, by the act of immunity, certain cells of the body become converted into cells that secrete specific antitoxin, and, as shown by Salmonson and Madsen, the administration of pilocarpin (which augments the secretion of most glands), also produces in immunized animals a rapid increase in the anti-toxin contents of their serum." This should give us pause, for here is laid down, in terms of the biologic laboratory, the precise method of action of this drug, which fortifies most of the cellular tissue of the body. This also explains its unusual power over many phases of disordered metabolism that often manifest themselves in ocular disease.

The brief recital of a few cases treated with pilocarpin may prove of interest:

C. A. R., female, at. 35, presents with an old central plastic choroiditis in the left eye, and beginning central choroiditis in the right eye, sharply confined to the macular region, and bordered all round with a fine vascular fringe. Vision with R.E. equalled 2/60. There were no clinical evidences of syphilis, and the Wassermann was negative. Heart and lungs were normal. Clinical history and findings were negative throughout. She was placed at once upon daily sweats with pilocarpin hypodermically, beginning at $1 / 20$ of a grain, and in two weeks increasing to $1 / 4$ of a grain. Salicylates were used freely without the slightest gastric irritability. Strychnia in ascending doses was given hypodermically daily up to $1 / 8$ of a grain. In three weeks the choroidal lesion in the right eye was absolutely quiet, and the vision had risen to $6 / 15$. To-day, after eight years, it is $6 / 12$, the choroidal lesion being still quiet, characteristic of the picture known as "hole in the macula."

M. I., female, age 25 , with a lesion in both maculæ corresponding to the right one in the preceding case. Wassermann positive. Heart, lungs, and kidneys normal. Placed on pilocarpin treatment, combined with vigorous mercurial treatment. Vision in both eyes when treatment was instituted $6 / 60$. One of the noteworthy features in connection with pilocarpin diaphoresis is the gigantic doses of any and all drugs that are tolerated without the slightest signs of toxæmia.

* W. B. Saunders \& Co., 1914. 
The biniodid of mercury in this case was carried up to $1 / 2$ grain three times a day. In three weeks, the vision in each eye equalled $6 / 9$, and after seven years, remains at that point. This case to-day also presents in each eye the typical picture of "hole at the macula."

F. E., male, cet. 40, single, the subject of cerebrospinal syphilis with bilateral complete iridoplegia and bilateral abducens palsy. Had been treated for a year with mixed treatment. Placed on pilocarpin diaphoresis daily, with biniodide pushed rapidly up to $1 / 2$ grain three times a day. In six weeks complete restoration and function of both external recti, with binocular vision.

K. J., male, at. 35, bilateral abducens palsy of two months' duration. Wassermann positive. Had been taking mercury and iodides steadily for two months with much intolerance. Heart, lungs, and kidneys normal. Pilocarpin diaphoresis, forced mercury and iodides, and ascending doses of strychnia hypodermically. In three weeks complete functioning of both external recti.

In these last two cases the chief value of the pilocarpin diaphoresis was as an adjuvant to the other medication.

N. $Z$., male, et. 45 , with typical retinitis pigmentosa. Had been told three weeks previously that his case was hopeless, and that he would eventually go blind surely. Had always been a very small drinker, but the dismal prognosis held out had so depressed him that he went on a week's debauch, at the end of which time he presented in my service at the Samaritan Hospital with vision of $3 / 60$ in each eye. He had the card of another hospital on which the recorded vision, before he went on his debauch, was 6/60 in each eye. His heart and kidneys were none too good, in spite of which, however, he was placed on pilocarpin diaphoresis and ascending doses of strychnia. At the end of four weeks his corrected vision was $6 / 12$, and this was held for five years, during all of which time he has been steadily at work, and supporting his family of five. I am far from holding that so dismal a condition as retinitis pigmentosa (which I take to be congenital) is curable. I simply quote this one case as illustrating the results of thoroughgoing therapeusis as against engendering in the mind of the patient the idea that he is doomed.

Mrs. C., et. 30, presents with a history of increasing exophthalmus for two weeks, with gradual impairment of motility and loss of sight. At the time she was first seen, the right eye was proptosed $13 \mathrm{~mm}$., displaced outward and downward, absolutely immobile, with vision equal to light perception only. There was high grade chemosis of the bulbar and palpebral conjunctiva. There had been frightful pain in the right fifth nerve distribution, which had given way to anæsthesia. The right pupil showed no direct nor consensual reaction; optic nerve normal. Retinal veins very full and tortuous. 
Optic nerve slightly œdematous. Mental depression was profound. The other eye was normal. There was a history of a nodule removed from the left breast four years previously, which had been pronounced by one of our good pathologists as malignant. This furnished a prognosis gloomy enough to suit the deepest-dyed pessimist. The Wassermann was negative, but this time we went over the head of the serologist and ordered mercurial inunctions and pilocarpin sweats. Within a week there was some slight improvement. In two weeks the motility of the eye began to return and sodium cacodylate intramuscularly was begun. In another fortnight motility in the eye was full and complete and the globe had receded to its normal position in the orbit. Unfortunately, the optic nerve had been too long pressed upon and it developed complete atrophy. But the woman meanwhile had put on 25 pounds, and to-day, after one year, is an active sound woman. The case has been detailed not to point out any special efficacy of pilocarpin, but simply to drive home the lesson that when we are licensed to practise and therapeutic weapons are placed in our hands, we solemnly engage to wage war upon disease and impending death to the uttermost, no matter how dark the outlook.

The use of pilocarpin by the mouth for its general lymphagogue properties has been urged by de Schweinitz for many years. He is in the habit of using $1 / 40$ to $1 / 20$ of a grain three times a day. In a patient over 45 with presenile arteriosclerosis and low-grade changes in the choroid and vitreous, there is no more potent agent than pilocarpin by the mouth. It reduces the vascular pressure, establishes better metabolism, equalises the circulation, and enhances the condition of such patients in every way. Idiosyncrasies must be watched for, as some patients will not tolerate more than $1 / 50$ or even $1 / 100$ of a grain three times a day.

With all the arguments against pilocarpin properly presented, I am still persuaded that by the hypodermic use of this drug, useful vision has been preserved to many patients, a result that would not have ensued with conventional methods. That the drug and its method of use deserve study and close observation goes without argument, but that it is one of the most potent weapons, particularly in the most obscure forms of intra- and extra-ocular disease is one of the convictions that have crystallized out of 25 years of experience in ocular therapeutics.

Salvarsan and neosalvarsan have become integral members of this family. In the presence of the positive Wassermann reaction they are strongly indicated, especially in disorders affecting the uveal tract. This is their field of brilliant efficiency. In syphilitic disorders of the optic nerve we must sometimes consider carefully before advising the organic arsenic compounds. In acute forms of the disorder we are disposed to recommend these agents if the 
laboratory indications are positive. But in the subacute and chronic forms we should vote for mercury by the mouth. In interstitial keratitis, the results of arsenobenzol medication have not fulfilled our expectations. On the contrary, our faith is pinned to mercury in the management of spirochetic parenchymatous keratitis. Nothing is more interesting than the return of our prominent syphilologists to mercury as the control of syphilis. Seven years ago we ventured the prophecy before the State Medical Society of Pennsylvania that the profession would eventually return to our good old friend Mercury as an antidote to Venus and her maladies, and we are to-day witnessing that very trend among our most advanced thinkers in syphilology.

The position of the salicylates is assured and requires no discussion.

\section{Pre- and Post-operative Treatment of Surgical Cases.}

This is a phase of ocular therapeutics that, in our judgment, is often slighted or, indeed, entirely neglected. If a glaucoma iridectomy for anything but the fulminant form of acute glaucoma is in prospect, a thoroughgoing course of the best possible elimination is strongly indicated. This is to be determined according to the caprice of the operator. "Chacun $\dot{a}$ son gout." Our own practice is to employ the formula of the late Dr. Goodell, namely, iron, quinine, and strychnia and mercury, with enough of the mercury to counteract the constipating effect of the iron in the Blaud's mass. Salines are used if needed, the preference being given to Sal Rochelle. A few pilocarpin sweats will be helpful in nearly every case. Miotics should be pushed vigorously up to the time of operation. The same procedure, with the exception of the miotics, is in order if extraction of cataract is contemplated. Argyrol is instilled into the eye after operation, as has been already outlined. The fourth day after the operation the iron, quinine, and strychnia and mercury combination is again instituted, with Sal Rochelle, as indicated. After cataract extraction, dionin is begun on the eighth day, to promote absorption of post-operative débris. This treatment is continuous until the fourteenth day after the operation. In patients over 50 years of age, there are few indeed who come to intra-ocular operations that are not the subjects of metabolic vices in the blood that may either lead or predispose to all manner of post-operative complications. As to complicated cataracts, that surgeon is bold indeed who will approach such operations without the most careful preparation of the patient for a fortnight in advance, and the same principles apply to the post-operative treatment of the case. Every surgeon of experience knows how small a percentage of cataract cases escape without a low post-operative iritis. Call it "quiet iritis," or what you will, we cannot blink the fact that it is 
almost bound to occur and may require but a small change in the quality of the blood stream to determine a nasty active iritis. To prevent such a disappointment after a skilfully performed operation, to turn an impending defeat into a victory, is one's simple duty, not only for the patient's sake but for the surgeon's also.

\section{Hormone- or Organo-Therapy.}

The introduction into therapeutics of preparations containing the active principles of the internal secretions is one of the achievements of modern medicine. In the blood there are countless substances of the nature of hormones, related to one another, and performing innumerable functions. Naturally, there is still a tendency to look askance upon the use of animal extracts in therapeutics. The attitude of the profession is well set forth in the following sentence from the preface of the work on the "Internal Secretions" by my colleague, Dr. Sajous. "If any daring member has introduced a subject bearing on medical treatment, it has been with an apologetic air or humble mien, well knowing that if his remarks had reference to the utility of drugs in the treatment of disease, they would be subjected to good humoured banter and received by those sitting in the seats of the scornful with amused incredulity."

Let us admit at the outset that the only valid test at present in the case of organic extracts is clinical experience under conditions which can be repeated by others with comparatively similar results. With this frank admission that all hormone therapy is as yet empirical ${ }^{*}$ in the best sense of that word, let us proceed to consider the preparations that may appeal to the ophthalmologist.

First and foremost, stand the preparations of the adrenal glands. Used locally in the eye, their action is now well understood. Their main application, in the very nature of things, is in ophthalmic surgery to obtain a bloodless ficld, for which purpose we are under a heavy debt to organotherapy, if for no other. But adrenalin and its congeners have other uses than these. In the treatment of exophthalmic goitre adrenalin substance is in high esteem by some of our best clinicians. Gibson at the 1912 meeting of the British Medical Association, urged that under its use the pulse rate was reduced, the protrusion of the eyeballs was markedly lessened, the thyroid diminished in size, and the tremor and other nervous symptoms vanished. We have seen the same gratifying results follow the use of thymus substance, and Harrower in his work ("Hormone Therapy") quotes a number of such observations.

* Since the presentation of this address, the application of the various Abderhalden tests for the specific ferments in the blood has been demonstrated, so that we may soon be upon more nearly scientific ground in determining what form our organotherapy shall take. 
Adrenalin solutions also have a certain value, perhaps, by altering intra-ocular pressure in conical cornea, as we have proven to our own satisfaction. And in those follicular conjunctival conditions that closely stimulate trachoma the combination of adrenalin and holocain, as suggested by Shoemaker, of St. Louis, has rendered us good service.

Thyroid substance naturally occupies a prominent place in this list of drugs. As pointed out by Radcliffe, the thyroid insufficiencies seen in women between 45 and 55 respond beautifully to thyroid medication, and many obscure ocular symptoms encountered in such subjects yield in a way that compels our attention. Another field of usefulness is in those forms of parenchymatous keratitis seen in husky young women when antisyphilitic medication has been unavailing. Especially in the sclerosing varieties of keratitis has it furnished brilliant results. But it is powerfully destructive in its metabolic effects, and should not for this reason be used in tuberculous subjects. The tendency has been to run too large doses. $1 / 5$ to $1 / 2$ a grain of thyroid substance is quite sufficient as a beginning dose.

About a year ago I mentioned in the New York Medical Journal a case of hypophyseal disease in a man of 35 with complete bitemporal hemianopsia. The hypophysis had in all probability gone into a state of cystic degeneration, as indicated by the skiagraph. Combined use of thyroid and pituitary substance brought about complete restoration of form and colour fields. This lasted about five years. Finally, the patient grew tired of the medication and discontinued it. In about a year he again lost both temporal fields absolutely. Again, he was placed on this combined organo-therapy, and, again, he regained full form and colour fields Once more, he grew negligent about his medication, and about six months ago, his temporal fields began to shrink. He disregarded this warning, and only recently has he resumed the hormone therapy. At the present writing his hemianopsia is complete even for light, and combined thyroid and pituitary medication is being forced. What the result will be this time, only the future can tell, but the results in this one case strongly indicate what may possibly happen in the future if such cases are taken early, and patients are made to understand that the organo-therapy will have to be adhered to. The mortality in these cases after operation is still too great to justify operative interference until hormone therapy has been fully tried.

Within two months a combination of thymus and pituitary substances has been used in an eleven-year old boy with classic interstitial keratitis, as an adjunct to the mercurial treatment. The boy was a stunted physical specimen, and the way he has developed physically is something to behold. In this same connection I cannot forbear mentioning two children who were brought to me for 
correction of errors of refraction. Both were perfectly formed children of high-grade mentality, but both were 2 to 3 years retarded in their physical development. In each case the thymuspituitary combination was suggested to and adopted by the family medical adviser, and, as in the case just recited, the response in physical growth was simply astonishing. In one case the accession in general physical well-being was so marked that the glasses were thrown aside.

A few words as to those post-operative asthenopias seen in women after oöphorectomies may not be amiss. That they try our uttermost resource, and are oft-times a pest, is a matter of general agreement. In three such cases within the past year, all the amazing ocular symptoms were dispelled by the constant use of ovarian extract. In dismissing this phase of ocular therapeutics, we can only record our conviction that the results to be obtained in this field are as yet undreamed of.

\section{Vaccine and Serum Therapy.}

If the charge of empiricism may be laid against much of our therapeutics, then here is a field in which one may be either the rankest empiricist or may adjust his therapy with the utmost scientific nicety. That the use of the tuberculin preparations is empirical cannot be gainsaid. And yet such an avalanche of evidence has accumulated that one cannot claim to be versed in ocular therapeutics if he do not stand ready to apply this remedy in suitable cases. Observe that I specify suitable cases. There is no class of cases that needs closer study for a correct final diagnosis than this peculiar class, and I ally myself whole-heartedly with those who claim that the diagnosis of ocular tuberculosis should not be made until every other possible causative factor has been ruled out. Once this has been done, tuberculin treatment may be instituted with due caution as to a minimal beginning dose, and care that the drug be kept within the point of pronounced reaction. In phlyctenular children, in episcleritis, in choroiditis, and in certain phases of iritis, there is no longer any doubting the beneficial action of tuberculin. To-day this is one of the best accepted clinical facts. Perhaps in the next few years we shall come upon more certain methods of diagnosing ocular tuberculosis. Besredka has recently succeeded in securing complement fixation reactions from the blood of tuberculous patients, and this may prove to be the way out of our difficulty, for in the past two years we have ourselves seen brilliant results follow vaccine therapy based on complement fixation reactions. In the Ophthalmic Record for January, 1915, Granville Lawrence and I have recorded three cases of gonorrhœal iritis in which the ætiologic diagnosis was made possible only by the complement fixation test for gonococcus. In each one of these cases there was absolutely no dis- 
coverable clinical evidence of gonorrhœa. In one case the gonorrhœal infection had occurred 15 years previously, in one case 5 years prior, to the iritis; and in one (a grandmother) we could get no history of a gonorrhœal infection. All threewere promptly treated with gonococcus vaccine, and that only, immediatelyon receipt of the serologist's report; and the relief of pain and clearing up of the iritis was little short of magic. In the November, 1915, number of the Ophthalmic Record I have put on record a case of influenzal iritis occurring in a 32 year old married man. The complement fixation test for spirocheta, gonococcus, pneumococcus, streptococcus, and staphylococcus was negative. There was a faint positive reaction for influenza, and on this finding, influenza vaccine was made the sole general treatment. The relief of pain was practically immediate ( 3 hours), and the inflammatory condition subsided in six days. Three weeks later there was a violent recurrence of a spongy iritis with an exudative membrane covering the whole iris and anterior capsule, and extending into the angle of the anterior chamber. Another injection of influenza bacterin was made, with prompt relief from pain, and complete disappearance of the membranous exudate in five days. The relief from the frightful fifth nerve pain that all such patients are subject to, 1s comparable only to the action of morphia. Within the past fortnight we have studied a plastic iritis in a 51 year old man, all of whose complement fixation tests were negative. His teeth were in a fearful condition, and complete extraction of all of them was advised by a dental surgeon after skiagraphic studies of the alveolar processes. The man was also given a mixed staphylococcus vaccine after bacteriologic study of his oral mucosa. When first seen, the pupil was $2.5 \mathrm{~mm}$., irregular, and bound-down all round. In ten days the eye was absolutely quiet, the media were all clear, and the corrected vision was $6 / 6$, as against $3 / 60$ when he was first seen. This was a pyorrhœal iritis, and the therapy was based on accurate diagnosis.

And now a few words as to vaccine therapy when no accurate indication is given by the complement fixation tests; that is to say, when it is based on clinical findings alone. Assume that one has to do with an iritis in a patient who has also been the subject of a polyarthritis. Even with all complement fixation tests negative, I do not think we need hesitate to use a streptococcic mixed vaccine in such a case. The amount of protein poison in such an injection does not seem to be able to do any harm, and the chances for resulting good are so large that we are justified in giving the patient the benefit of the doubt. Let us admit that vaccines are protein poisons of low degree potency. Let us admit, further, that the treatment is based on clinical findings only. Do not the gentlemen who treat pneumonia and gastric ulcer and appendicitis on a clinical basis, pure and simple, fill their patients with drugs that are low-grade 
poisons, just as hopefully as if they administered a vaccine on the same grounds? Hundreds of thousands of vaccine injections have been administered in this country in the last five years, and few indeed are the reports that have come to hand of any deleterious effects.

In chronic dacryocystitis a mixed vaccine of streptococcus and staphylococcus has rendered us valuable service as an adjunct to the local treatment, and in a threatened post-operative infection after cataract extraction, an eye that gave every promise of going to the bad was saved by sero-bacterins.

I am far indeed from advocating the indiscriminate use of vaccines. It is the greatest danger with which our profession is just now threatened. We cannot, therefore, insist too much on the search for the most accurate findings possible on which to formulate working conditions. Nevertheless, I hold that when the laboratory leaves us in doubt, we are justified in searching for clinical facts to justify the employment of these newer weapons that promise so much to gravely threatened eyes.

What has been said may reveal what many will consider a bias in favour of vaccine therapy. When the laboratory findings are definite, we unhesitatingly endorse vaccine treatment, and so far as properly selected clinical cases are concerned, we are again perfectly willing to admit the soft impeachment. Let us, in the words of St. Paul, "Prove all things; and hold fast to that which is good."

If certain phases of ocular therapeutics have been herein omitted, it was because of my feeling that personal experience would find more favour than a mere review of the present status of ocular therapeutics in general.

In conclusion, let us not be either over enthusiastic about the qualities of the new, nor iconoclastic regarding the therapeutic ideals of olden days. Let us rather cultivate the diagnostic sixth sense, the faculty of hewing close to the line, and add to these, if we may, the rare gift of separating the few grains of fact from the dust of uncertainty that has enveloped therapeutics in general for the past quarter of a century. 